\title{
Flow-Diverter Stents for the Treatment of Saccular Middle Cerebral Artery Bifurcation Aneurysms: Is "Unsuitable" the Right Conclusion?
}

W e read with interest the recent publication by Caroff et $\mathrm{al}^{1}$ on saccular MCA aneurysm treatment by flow diverters (FDs), in regard to the increasing tendency to use FDs in distal, off-label localizations. We appreciate their important contribution to the subject but remain reserved regarding their strong conclusion. The subject is still under research, but some promising clinical and angiographic outcomes have been published. ${ }^{2} \mathrm{We}$ were slightly perplexed by the interpretation of the reported complication rate, taking into account the methodologic limitations of the study due to the small number of subjects.

Various mechanisms of ischemic complications are implicated in flow diversion with covered arteries, which are not always sufficiently represented in a small case series. Thrombosis related to an ineffective anti-aggregation regimen, suboptimal stent apposition on the arterial wall, or distal emboli provoked by the mechanical manipulations inside the arteries are types of complications that should not be confounded with the pure hemodynamic effect of flow diversion on a covered branch. Good discrimination of the various potential ischemic mechanisms is mandatory to obtain a more profound understanding of FD-related complications.

To clear a misunderstanding, we must comment on the complication rates of the case series by Saleme et $\mathrm{al}^{3}$ cited in the discussion. The overall complication rate for the MCA cases in the study was 5\% ( $n=1 / 19)$, with a sole side branch-related complication in the MCA aneurysm sub-series. Apart from reporting complication rates, they performed a classification of jailed branches, dividing them into anastomotic or terminal. In this section, a symptomatic angiographic change was defined as any angiographic remodelling at 6 months in patients who reported transient symptoms at any point during the follow-up of 6 months.

These findings, reported in the postprocedural period analysis, usually occurred around 3-4 weeks after stent placement and consisted of either transient symptoms or silent DWI spots. They were reported to delineate the progressive remodelling of jailed branches, as part of the collateral network development of the brain ${ }^{4}$ in the postprocedural period. They were described in patients under effective antiaggregation and should not be confounded with early ischemic findings in patients with inefficient antiaggregation or with immediate flow restriction in the jailed branches, related to local high metal coverage or stent-deployment defects.

The complications reported in the study of Caroff et $\mathrm{al}^{1}$ were encountered in the immediate postprocedural period; as they mentioned, the complications seemed to be related mainly to antiaggregation and technical issues. These results are useful to plan further, larger scale studies; they should also be kept in mind in everyday practice. Controlled hypertension in the postprocedural period, avoidance of under- or overinhibition of P2Y12 receptors, ${ }^{2}$ and slight device oversizing are key technical points to consider when covering MCA branches.

The use of FDs in MCA bifurcations is still under research; various parameters need to be clarified and standardized before establishing a consensus for this treatment. Jailing distal terminal intracranial branches such as MCA bifurcations remains a challenging issue, but with a careful hemodynamic case-by-case study and proper patient preparation, FD treatment in such cases may prove to be a useful technique in otherwise challenging complex cases. Further research is underway regarding this subject by several teams; until concrete data see the light, every conclusion should be taken with a grain of salt.

\section{REFERENCES}

1. Caroff J, Neki H, Mihalea C, et al. Flow-diverter stents for the treatment of saccular middle cerebral artery bifurcation aneurysms. AJNR Am J Neuroradiol 2015 Sep 24. [Epub ahead of print] CrossRef Medline

2. Kallmes DF, Hanel R, Lopes D, et al. International retrospective study of the Pipeline embolization device: a multicenter aneurysm treatment study. AJNR Am J Neuroradiol 2015;36:108-15 CrossRef Medline

3. Saleme S, Iosif C, Ponomarjova S, et al. Flow-diverting stents for intracranial bifurcation aneurysm treatment. Neurosurgery 2014; 75:623-31; quiz 631 CrossRef Medline

4. Rouchaud A, Leclerc O, Benayoun $\mathrm{Y}$, et al. Visual outcomes with flow-diverter stents covering the ophthalmic artery for treatment of internal carotid artery aneurysms. AJNR Am J Neuroradiol 2015; 36:330-36 CrossRef Medline

(1). losif (1) C. Mounayer Department of Interventional Neuroradiology Dupuytren University Hospital Limoges, France 